\title{
On a mathematical model of dynamics of the elastic wedge-shaped medium with radiating defect
}

\author{
Vyacheslav Berkovich ${ }^{1, *}$, Dmitrii Pozharskii ${ }^{2}$, Victor Poltinnikov ${ }^{2}$, and Anna Morozova ${ }^{3}$ \\ ${ }^{1}$ Don Cossacks' State Institute of Food Industry and Business (brunch) «MGUTU» named after \\ K.G.Razumovsky (First Cossacks' University), 55, Semashko, 344007, Rostov-on-Don, Russia \\ ${ }^{2}$ Don State Technical University, 1, Gagarin sq., 344010, Rostov-on-Don, Russia \\ ${ }^{3}$ Rostov State Transport University, 2, Rostovskogo Strelkovogo Polka Narodnogo Opolcheniya sq., \\ 344038, Rostov-on-Don, Russia
}

\begin{abstract}
In the paper the mixed boundary value problem of antiplane vibrations is considered in the elastic wedge-shaped medium containing the radiating defect $J_{2}$. Radiating generators are assumed to be located on defect boundaries and on the interval $J_{1}$ of the wedge free boundary as well. The problem of reconstructing the wave field in the whole wedgeshaped region with its boundary is stated. A number of problems of analyzing acoustic emission signals by radiating defect are reduced to the problem considered in connections with using non-destructive testing elements of the technological equipment under exploitation. The problem in question is reduced to studying the solvability problems of the equivalent boundary integral equation system both for stress saltus on the defect $J_{2}$ and contact stresses on the interval $J_{1}$ of the upper plane of the wedge.
\end{abstract}

\section{Introduction}

The aim of the present paper is mathematical modeling of a pre-fracture state of the construction unit representing the junction of angular elastic elements. It is investigated correctness problems of applying mathematical modeling method for the wave process arising in angular elements examined by non-destructive testing methods both in hard industry enterprises and in ones of agricultural machinery. Under long dynamic exploitation of the technological equipment it appears the stress singularity at the angular point. In its neighborhood there arises the defect growing to the angular point (stress concentration) and generating the acoustic radiation (acoustic emission - AE). Non-destructive testing methods are worked out in details in [1-6]. At the paper the pre-fracture state is considered, provided the appearance of the radiating defect takes place only in one of angular elements. The angular element is modeling by elastic body of wedge-shaped medium, one of its planes is stiffly connected with other angular elements, radiating defect is modeling by the linear radial cut of finite length, antiplane vibration generators being located on the cut

\footnotetext{
*Corresponding author: bvn06@yandex.ru
} 
boundaries. The contact interaction zone is modeling by the harmonic oscillating punch under antiplane deformation (Fig.1).

1. The boundary value problem is formulated for the dynamic elasticity equations in the domain $\Omega$, presenting the angle of span $\alpha$ with cut $J_{2}$, simulating the defect located on the segment $\left[a_{2}, b_{2}\right]$ of the line $G_{\alpha_{0}}$. Oscillating coherent generators of the antiplane shear displacements $f_{2}(r) e^{-i \omega t}$ of the equal intensity are located on the banks of $J_{2}^{ \pm}$.

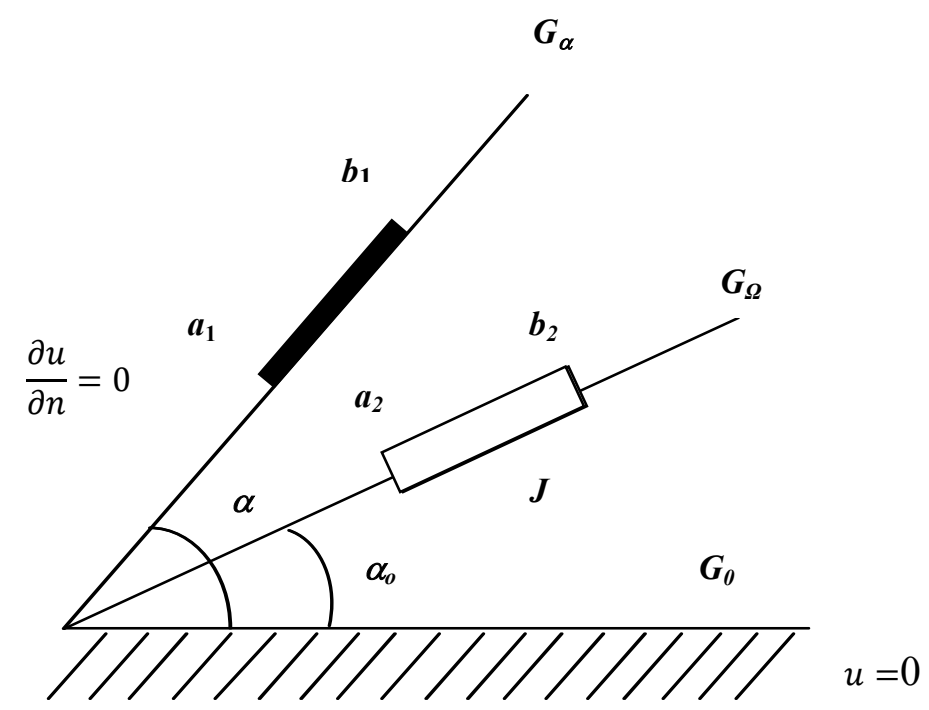

Fig. 1. Elastic wedge-shaped medium with radiating defect.

On the upper plane $G_{\alpha}$ harmonic oscillation generators $f_{1}(r) e^{-i \omega t}$ are located on the finite segment $\left[a_{1}, b_{1}\right]$ as well. The rest of the upper plane $G_{\alpha}$ is assumed to be unloaded and the lower plane $G_{0}$ is stiffly connected. In the vertex $r=0$ there are no radiation sources and Somerfield's radiation conditions take place at infinity. Under the steady oscillations there states the problem of finding the unknown contact stresses in the zone $J_{1}$ of the contact interaction with the punch, stress saltus on the cut $J_{2}$ and the reconstructing displacement field in the whole domain $\Omega$ including the upper boundary $G_{\alpha}$.

Under harmonic oscillations with the frequency $\omega$ displacements $U(r, \varphi, t)$ obey the dynamic elasticity equations and are sought in the form $U(r, \varphi, t)=u(r, \varphi) \exp (-i \omega t)$. The problem in question as it is known in the classic statement is reduced to the next boundary value problem for the Helmholtz equation of complex displacement amplitude $u(r, \varphi)$ :

$$
\begin{gathered}
\Delta u+K^{2} u=0, K^{2}=D \omega^{2} / \mu \\
\left.u\right|_{\varphi=\alpha}=f_{1}(r), r \in\left(a_{1}, b_{1}\right) \\
\left.\sigma_{r \varphi}\right|_{\varphi=\alpha}=0, r \notin\left(a_{1}, b_{1}\right) \\
\left.u\right|_{\varphi=0}=0, \quad u \mid J_{2}^{ \pm}=f_{2}(x, y), \quad(x, y) \in J_{2}^{ \pm},
\end{gathered}
$$

where $D, \mu$ are density and shear modules of the wedge material, $J_{2}^{ \pm}$is the left and right cut boundaries of the cut $J_{2}=J_{2}^{-} \cup J_{2}^{+}$respectively. No radiation sources are assumed to be on 
the vertex $r=0$, displacements vanish at infinity and Somerfield's radiation conditions principle take place:

$$
\frac{\partial u}{\partial r}-i K u=o\left(r^{-\frac{1}{2}}\right), r=\sqrt{x^{2}+y^{2}} \rightarrow \infty
$$

The lack of radiation sources on the vertex $r=0$ provides the fulfilment the SaintVenant principle and the existence of the solution in Sobolev space $W_{2}^{1}(\Omega)$, the norm being given by the traditional way.

Solving the problem stated above is based on its reducing to the equivalent boundary integral equations (BIE) about the unknown (dimensionless) contact stresses amplitude $\left.\mu^{-1} \sigma_{r \varphi}\right|_{\varphi=\alpha}=q_{1}(r), a_{1}<r<b_{1}$ on the upper plane and the unknown amplitude saltus of (dimensionless) stresses $\left.\left[\mu^{-1} \sigma_{r \varphi}\right]\right|_{\varphi=\alpha_{0}}=q_{2}(r), a_{2}<r<b_{2}$ on the $\operatorname{defect} J_{2}$.

The statement of the problem in question leads to the next boundary value problem in the domain $\Omega$. To solve the problem there fulfils the construction of Green function $G(r, \varphi \mid \rho, \psi)$ in the wedge-shaped domain without defect. Green function obeys the nonhomogeneous Helmholtz equation and boundary conditions as follows:

$$
\begin{gathered}
\Delta G+K^{2} G=-\frac{1}{4 \pi r} \delta(\rho-r) \delta(\psi-\varphi), K^{2}=D \omega^{2} / \mu \\
\left.\frac{\partial G}{\partial n}\right|_{\varphi=0}=\left.G\right|_{\varphi=\alpha}=0,
\end{gathered}
$$

where $\delta(x)$ is Dirac's function, $n$ is the external normal to the boundary. Green function method is worked out in details [7-9] when solving static problems. The same method permits to solve boundary value problems of the dynamic elasticity and reconstruct the wave field generated by all vibration sources in the whole wedge-shared medium considered. As in the statement of the main problem (1), (2) no radiation sources are assumed to be on the vertex $r=0$ and the same conditions at infinity take place.

To construct Green function obeying non-homogeneous Helmholtz equation and boundary conditions (4), (5) the Kontorovich-Lebedev integral transform methods are used in the form

$$
\begin{aligned}
\bar{f}(\tau) & =\int_{0}^{\infty} f(r) K_{-i \tau}(\kappa r) \frac{d r}{r} \\
f(r) & =\frac{1}{\pi i} \int_{-\infty}^{\infty} \bar{f}(\tau) I_{-i \tau}(\kappa r) \tau d \tau
\end{aligned}
$$

where $I_{v}(\kappa r), K_{v}(\kappa r)$ are modified Bessel functions.

Series of awkward transformations connected with solving the problem (4), (5) results the expression of Green function as follows:

$$
\begin{gathered}
G(r, \varphi \mid \rho, \psi)=\int_{0}^{\infty} \bar{G}(\tau, \varphi, \psi) K_{-i \tau}(\kappa r) I_{-i \tau}(\kappa \rho) \tau d \tau, \\
\bar{G}(\tau, \varphi, \psi)=\left\{\begin{array}{l}
2 \frac{\operatorname{sh} \psi \tau \operatorname{ch}(\alpha-\varphi) \tau}{\operatorname{ch} \alpha \tau}, \varphi>\psi, \\
2 \frac{\operatorname{sh} \varphi \tau \operatorname{ch}(\alpha-\psi) \tau}{\operatorname{ch} \alpha \tau}, \varphi<\psi
\end{array}\right.
\end{gathered}
$$


To obtain the boundary integral equation, to use the well-known integral representation of the regular solution of Helmholtz equation in the next form

$$
\begin{gathered}
u(x, y)=-\frac{1}{2 \pi} \int_{L_{R}}\left\{G(x, y \mid \xi, \eta) \frac{\partial u}{\partial n}-u(\xi, \eta) \frac{\partial}{\partial n} G(x, y \mid \xi, \eta)\right\} d l_{n} \\
L_{R}=G_{\alpha}^{R} \cup G_{0}^{R} \cup J_{2}^{+} \cup J_{2}^{-} \cup G_{R}, \quad J=J_{2}^{+} \cup J_{2}^{-}
\end{gathered}
$$

In the formulas (7) contour $G_{R}$ is the part of the circumference of the finite radius $R$ (with the center in the wedge vertex), closing the angular domain, $G_{\alpha}^{R}, G_{0}^{R}$ are parts of $G_{\alpha}, G_{0}$ truncated by $G_{R}, n$ is external normal to the boundary, $J_{2}^{ \pm}$are banks of the cut $J_{2}$. By virtue of coherence and equal intensity of vibration sources on the cut banks, boundary conditions, vanishing conditions for displacements and Green function as $R \rightarrow \infty$, as well as radiation conditions (3) we obtain the limit expression of displacement in the whole wedge-shaped domain $\Omega$ :

$$
u(x, y)=\frac{1}{2 \pi} \int_{J_{+}} G\left[\frac{\partial u}{\partial n}\right]_{J_{2}} d l_{n}-\left.\left.\frac{1}{2 \pi} \int_{a}^{b} G\right|_{\psi=\alpha} \frac{\partial u}{\partial n}\right|_{J_{1}} d l_{n}
$$

In (8) let observation point $(x, y)$ tends the left bank of the cut $J_{2}$, then let the latter tends to the segment $J_{1}$ of the upper wedge plane where vibration sources are given and pass to the polar coordinates $(r, \varphi)$. There results the boundary integral equation (BIE) system about the amplitude stress saltus $q_{2}$ on the cut $J_{2}$ and amplitude contact stresses $q_{1}$ on the segment $J_{1}$. BIE system takes the form:

$$
\begin{gathered}
\int_{a_{1}}^{b_{1}} k_{11}(r, \rho) q_{1}(\rho) d \rho+\int_{a_{2}}^{b_{2}} k_{12}(r, \rho) q_{2}(\rho) d \rho=f_{1}(r), \quad a_{1} \leq r \leq b_{1} \\
\int_{a_{1}}^{b_{1}} k_{21}(r, \rho) q_{1}(\rho) d \rho+\int_{a_{2}}^{b_{2}} k_{22}(r, \rho) q_{2}(\rho) d \rho=f_{2}(r), a_{2} \leq r \leq b_{2}
\end{gathered}
$$

Let us introduce matrixes as follows

$$
\begin{gathered}
k(r, \rho)=\left(\begin{array}{ll}
k_{11}(r, \rho) & k_{12}(r, \rho) \\
k_{21}(r, \rho) & k_{22}(r, \rho)
\end{array}\right), \\
k(r, \rho)=\frac{2}{\pi^{2}} \int_{0}^{\infty} K_{-i \tau}(\kappa \rho) K_{-i \tau}(\kappa r) k(\tau) \tau \operatorname{sh} \pi \tau d \tau, \kappa=-i K \\
k(\tau)=\left(\begin{array}{ll}
K_{11}(\tau) & K_{12}(\tau) \\
K_{21}(\tau) & K_{22}(\tau)
\end{array}\right)=\left(\begin{array}{cc}
\frac{\operatorname{th} \alpha \tau}{\tau} & \frac{\operatorname{sh} \alpha_{0} \tau}{\tau \operatorname{ch} \alpha \tau} \\
\frac{\operatorname{sh} \alpha_{0} \tau}{\tau \operatorname{ch} \alpha \tau} & \frac{\operatorname{sh} \alpha_{0} \tau \operatorname{ch}\left(\alpha-\alpha_{0}\right) \tau}{\tau \operatorname{ch} \alpha \tau}
\end{array}\right) .
\end{gathered}
$$

Let us consider the auxiliary BIE, constructed on the base of (9), (10) and written in the abbreviated vector-matrix form $\left(\begin{array}{c}a=\min _{i=1,2}, \quad b=\max _{i=1,2} b_{i}\end{array}\right)$ :

$$
\begin{gathered}
\int_{a}^{b} k(r, \rho) \bullet q(\rho) d \rho=\tilde{f}(r), a<r<b \\
\tilde{f}=\left(\begin{array}{c}
\tilde{f}_{1}(r) \\
\tilde{f}_{2}(r)
\end{array}\right), q=\left(\begin{array}{c}
q_{1}(\rho) \\
q_{2}(\rho)
\end{array}\right)
\end{gathered}
$$


In the correlation (11) $\tilde{f}_{1,2}(r)$ are results of extending of functions $f_{1,2}(r)$ to the interval $(a, b)$, matrix function $k(\tau)$ is real both on the imaginary axis and on the real one where $k(\tau)$ is positively defined.

2. To investigate the solvability problems for the BIE system the next theorem is established.

\section{Theorem}

Operator $K$ of the left hand side (11) is uniquely inverted as operator acting in vector function spaces:

$$
\begin{aligned}
& K: H(a, b) \rightarrow W_{2}^{\frac{1}{2}}(a, b) \\
& H(a, b) \subset W_{2}^{-\frac{1}{2}}(a, b)
\end{aligned}
$$

where $W_{2}^{\gamma}(a, b), \gamma= \pm 1 / 2$ are Sobolev-Slobodetsky spaces of fractional smoothness.

To prove the theorem, to put $\kappa>0$ temporary. Then by virtue of the positive definition of matrix $k(\tau), \tau \in R^{1}$ the operator $K$ appears to be positively defined as well and induces the space $H(a, b)$ of generalized solutions of the equation (11), one being introduced by the norm

$$
\begin{gathered}
\|q\|_{H(a, b)}=\left(\int_{0}^{\infty} Q^{T}(x) \cdot K(x) \cdot Q^{*}(x) d x\right)^{\frac{1}{2}} \\
Q(x)=\sqrt{x \operatorname{sh} \pi x} \int_{a}^{b} q(s) K_{-i x}(\kappa s) d s, \quad 0<x<\infty
\end{gathered}
$$

and the scalar product (* means complex conjugation, $T$ is the transposition option):

$$
\left(q_{1}, q_{2}\right)_{H}=\int_{0}^{\infty} Q_{1}^{T}(x) \cdot K(x) \cdot Q_{2}^{*}(x) d x
$$

The use of Riesz's theorem on uniqueness of representation of linear continual functional in the Hilbert space [5] adduces to the solvability condition for BIE system (11) as follows

$$
\begin{gathered}
M^{2}=\int_{0}^{\infty} F^{T}(x) \cdot K^{-1}(x) \cdot F^{*}(x) d x<\infty, \\
F(x)=\sqrt{x \operatorname{sh} \pi x} \int_{0}^{\infty} \tilde{f}(s) K_{-i x}(\kappa s) d s .
\end{gathered}
$$

Constructing the special two-sided estimation for the magnitude $M^{2}$ obtaining by the use of the integral representation of McDonald function $K_{-i x}(\kappa s)$ [4] and Parceval equality for Fourier integral transform adduce to the condition $\tilde{f} \in W_{2}^{\frac{1}{2}}(a, b)$. It is well-known the space $W_{2}^{-1 / 2}(a, b)$ is conjugate to the space $W_{2}^{1 / 2}(a, b)$ [6]. Then from the Riesz's theorem it points out both the existence of the unique solution $q \in W_{2}^{-1 / 2}(a, b)$ for any right hand side $\tilde{f} \in W_{2}^{\frac{1}{2}}(a, b)$ and the imbedding $H(a, b) \subset W_{2}^{-1 / 2}(a, b)$. 
The result described is in accordance with known results on boundary properties of functions belonging to Sobolev spaces $W_{2}^{1}(a, b)$ in which the solution of boundary value problems is searched by the dynamic elasticity methods.

The passage to the initial case $\kappa=-i k$ is provided by the analytical continuation principle [12] since all functions are analytical with respect to $\kappa$ in the domain $\operatorname{Re} \kappa \geq 0$, $\kappa \neq 0$ of the complex plane, where, in part, the point $\kappa=-i k$ is located.

It permits to ascertain the unique solvability of the initial boundary value problem (1), (2) in the Sobolev space $W_{2}^{1}(\Omega)$ for the whole wedge-shaped domain and there results the inequality :

$$
\|q\|_{H(a, b)} \leq \mathrm{C}\|\tilde{f}\|_{W_{2}^{\frac{1}{2}(a, b)}}, C=\text { const }
$$

meaning the correct solvability of the problem in question permitting to apply varies analytical (for example, methods in[12]) and numerical methods to approach sought-forfunctions $q_{1}, q_{2}$ as solutions of the BIE system (9), (10).

The consequent use of described results to narrowing of functions $q_{i}, f_{i}(i=1,2)$ from the domain $(a, b)$ to the initial ones $\left(a_{i}, b_{i}\right) \subset(a, b)$ leads to the unique resolution of the initial BIE system (9), (10) in spaces of the fractional smoothness. It means there exists the unique solution $q_{i} \in W_{2}^{\frac{-1}{2}}\left(a_{i}, b_{i}\right)$ for any right hand side function

$$
f_{i} \in W_{2}^{\frac{1}{2}}\left(a_{i}, b_{i}\right), i=1,2
$$

The reconstruction of displacement wave field in $\Omega$ and in the boundary $G_{\alpha}$ may be fulfilled by representation (8) which is presented by means of displacements' amplitude $f_{2}(x, y)$ when radiating $\mathrm{AE}$ from defect boundaries. The displacement wave field may be considered as the base to the statement of the inverse problem of reconstructing the displacements' amplitude $f_{2}(r)$ on the defect $J_{2}$ by means of direct displacement measurements. It may be done when constructing the displacements' amplitude frequency reply on the unloaded part of the boundary $G_{\alpha}$ and the sequel application of the least square method $[14,15]$.

\section{References}

1. S.I. Builo, Physical, mechanical Statistical and chemical aspects of acoustic emission diagnostics (Southern Federal University Press, Rostov-on-Don, 2017)

2. V.I. Ivanov, V.A. Barat, Acoustic emission diagnostics (Publishing «Spectr», Moscow, 2017)

3. S.I. Builo, Russ. J. Nondestr. Test. 54(4), 237-242 (2018)

4. S.I. Builo, Russian Journal of Nondestuctive Testing 11, 32-45 (2012)

5. S.I. Builo, Physics and Mechanics of New Materials and Their Applications N(ova Science Publishers, New York, 2013)

6. S.I. Builo, D.M. Kuznetsov, V.L. Gaponov, Russian Journal of Nondestructive Testing 55(11), 803-807 (2019)

7. M.Yu. Kashtalyan, J.J. Rushchitsry, Int. Appl. Mech. 46(1), 1-17 (2010)

8. D.A. Pozharskii, J. Appl. Math. and Mech. 80(4), 489-495 (2018)

9. B. Sobol, A. Krasnoschekov, A. Soloviev, ZAMM 95(11), 1302-1314 (2015)

10. E. Pan, W. Chen, Static Green's function in anisotropic media (Cambridge University 
Press, New York, 2015)

11. Y. Xu, R.L. Jackson, J. Tribology 140(1), 1401-1404 (2018)

12. V.N. Berkovich, A.V. Babkin, Int. Res. J. 58(23), 101-104 (2017) DOI: http//doi.org/10.23670/IRJ. 2017.58.042.

13. V.N. Berkovich, Int. Res. J. 2(92), 19-24 (2020) DOI: http//doi.org/10.23670/IRJ.2020.92.2.003

14. R. Nedin, A. Vatulyan, Int. J. Solids Struct. 50, 2107-2114 (2013)

15. V.N. Berkovich, S.I. Builo, Russian Journal of Nondestuctive Testing 4, 262-267 (2019) 